Acta Phys. Hung. A 25/1 (2006) 000-000

HEAVY ION

PHYSICS

\title{
Quantum Clan Model description of Bose-Einstein correlations
}

\author{
O.V.Utyuzh ${ }^{1, a}$, G.Wilk ${ }^{1, b}$ and Z.Włodarczyk ${ }^{2}$ \\ 1 The Andrzej Soltan Institute for Nuclear Studies; \\ Hoża 69; 00-681 Warsaw, Poland \\ 2 Institute of Physics, Świętokrzyska Academy, \\ Świętokrzyska 15; 25-406 Kielce, Poland; E-mail: wlod@pu.kielce.pl \\ ${ }^{a}$ E-mail: utyuzh@fuw.edu.pl \\ ${ }^{b}$ E-mail: wilk@fuw.edu.pl
}

Received 7 May 2005

\begin{abstract}
We propose a novel numerical method of modelling Bose-Einstein correlations (BEC) observed among identical (bosonic) particles produced in multiparticle production reactions. We argue that the most natural approach is to work directly in the momentum space in which the Bose statistics of secondaries reveals itself in their tendency to bunch in a specific way in the available phase space. Because such procedure is essentially identical to the clan model of multiparticle distributions proposed some time ago, therefore we call it the Quantum Clan Model.
\end{abstract}

Keywords: Bose-Einstein correlations; Statistical models; Fluctuations PACS: 25.75.Gz 12.40.Ee 03.65.-w 05.30.Jp

The phenomenon of Bose-Einstein correlations (BEC) is so widely known [1] that we shall start directly with problem of its proper numerical modelling, such which would account from the very beginning for the quantum statistical bosonic character of identical secondaries produced in hadronization process. To our knowledge this problem was so far considered only in [ 2] (cf., however, [ 3]). All other approaches claiming to model BEC numerically [4] use as their starting point the outcomes of existing Monte-Carlo event generators (MCG) describing multiparticle production process [ [5] and modify them in a suitable way to fit the BEC data. These modifications are called afterburners. They inevitably lead to such unwanted features as violation of energy-momentum conservation or to changes in the original multiparticle spectra. Actually, it is worth to mention at this point that in [ [6] we proposed afterburner free from such unwanted effects. It was based on different concept of introducing quantum mechanical (QM) effects in the otherwise purely 
probabilistic distributions from those proposed in [7]. Namely, each MCG provides us usually with a given number of particles, each one endowed with one of $(+/-/ 0)$ charges and with well defined spatio-temporal position and energy-momentum. On the other hand, experiment provides us information on only the first and last characteristics. The spatio-temporal information is not available directly (in fact, the universal hope expressed in [1, 4] is it can be deduced from the previous two via the measured BEC). Our reasoning was as follows: $i$ ) BEC phenomenon is of the QM origin, therefore one has to introduce in the otherwise purely classical distributions provided by MCG a new element mimicking QM uncertainties; ii) this cannot be done with energy-momenta because they are measured and therefore fixed; iii) the next candidate, i.e. spatio-temporal characteristics, can be changed but this was already done in [ 7, 4]; iv) one is thus left with charges and in [6] we simply assigned (on event-by-event basis) new charges to the particles selected by MCG conserving, however, the original multiplicity of $(+/-/ 0)$. This has been done in such a way as to make particles of the same charge to be located maximally near to each other in the phase space by exploring natural fluctuations of spatio-temporal and energy-momentum characteristic resulting from MCG. In this way we automatically conserve all energy-momenta and do not change multiparticle distributions and do it already on the level of single event provided by MCG. However, the new assignment of charges introduces a profound change in the structure of the original MCG. Generally speaking (cf. [ [6] for details), it is equivalent to introduce the bunching of particles of the same charge used in the MCG.

This observation will be the cornerstone of our new proposition. Let us remind that idea of bunching of particles as quantum statistical (QS) effect is not new [ 8]. It was used in connection with BEC for the first time in [ 9] and later it was a cornerstone of the clan model of multiparticle distributions $P(n)$ leading in natural way to their negative binomial (NB) form observed in experiment [10]. It was introduced in the realm of BEC in [11] and [2, 3. Because our motivation comes basically from [2] let us outline shortly its basic points. It deals with the problem of how to distribute a given number of bosonic secondaries, $\langle n\rangle=\left\langle n^{(+)}\right\rangle+$ $\left\langle n^{(-)}\right\rangle+\left\langle n^{(0)}\right\rangle,\left\langle n^{(+)}\right\rangle=\left\langle n^{(-)}\right\rangle=\left\langle n^{(0)}\right\rangle$, in a least biased way . Using information theory approach (cf., [12]) their rapidity distribution was obtained in form of grand partition function with temperature $T$ and chemical potential $\mu$. In addition, the rapidity space was divided into cells of size $\delta y$ (fitted parameter) each. It turned out that whereas the very fact of existence of such cells was enough to obtain reasonably good multiparticle distributions, $P(n)$, (actually, in the NB-like form), their size, $\delta y$, was crucial for obtaining the characteristic form of the 2-body BEC function $C_{2}\left(Q=\left|p_{i}-p_{j}\right|\right)$ (peaked and greater than unity at $Q=0$ and then decreasing in a characteristic way towards $C_{2}=1$ for large values of $Q$ ) out of which one usually deduces the spatio-temporal characteristics of the hadronization source [1] (see [ 2 for more details). The outcome was obvious: to get $C_{2}$ peaked and greater than unity at $Q=0$ and then decreasing in a characteristic way towards $C_{2}=1$ for large values of $Q$ one must have particles located in cells in phase space which are of nonzero size. ${ }^{a}$ 


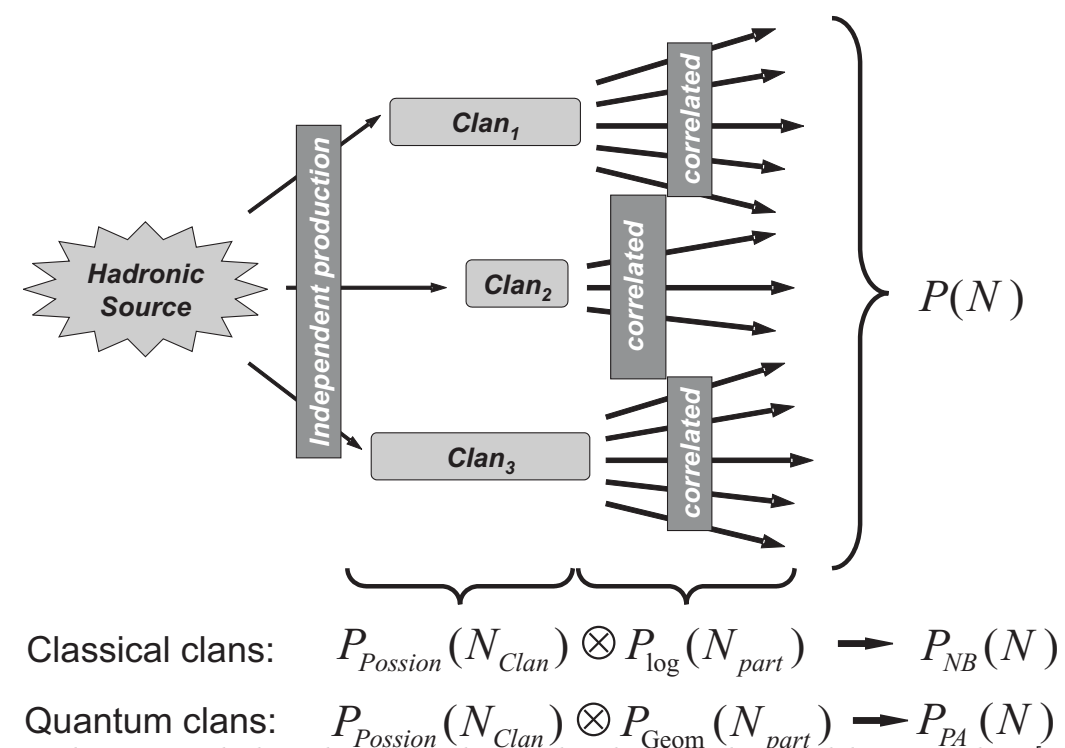

Fig. 1. The proposed algorithm is similar to the classical clan model proposed in [10] but its clans contain particles of the same charge and (almost) the same energies and are distributed according to geometrical distribution what results in overall Pòlya-Aeppli distribution, $P_{P A}(n)$ [ 16 , insted of NB one, $P_{N B}(n)$.

To illustrate our proposition let us assume that mass $M$ hadronizes into $N=\langle n\rangle$ bosonic particles (we take them as pions of mass $m$ ) with equal numbers of $(+/-/ 0)$ charges and with limited transverse momenta $p_{T}$. Suppose that their multiplicity distribution $P(n)$ follows a NB-like form (i.e. it is broader than Poissonian) and that their two-particle correlation function of identical particles, $C_{2}(Q)$, has the specific BEC form mentioned above. To model such process accounting from the very beginning, for the bosonic character of produced particles we propose the following steps (illustrated by comparison to some selected LEP $e^{+} e^{-}$data [17, cf., Fig. 1):

1) Using some (assumed) function $f(E)$ select a particle of energy $E_{1}^{(1)}$ and charge $Q^{(1)}$. The actual form of $f(E)$ should reflect somehow our a priori knowledge of the particular collision process under consideration. In what follows we shall assume that $f(E)=\exp (-E / T)$, with $T$ being parameter (playing in our example the role of "temperature").

2) Treat this particle as seed of the first elementary emitting cell (EEC) (introduced in [11]) and add to it, until the first failure, other particles of the same charge $Q^{(1)}$ selected according to distribution $P(E)=P_{0} \cdot \exp (-E / T)$, where $P_{0}$ is another parameter (playing the role of "chemical potential" $\mu=T \cdot \ln P_{0}$ ). This assures that the number of particles in this EEC, $k_{1}$, will follow geometrical (or Bose-Einstein) distribution, i.e. $\left\langle k_{1}\right\rangle=P(E) /[1+P(E)]$, and accounts therefore for their bosonic character. As result $C_{2}(Q)>1$ but only at one point, namely for 3) Iø.get the experimentally observed width of $C_{2}(Q)$ one has to allow that parti- 

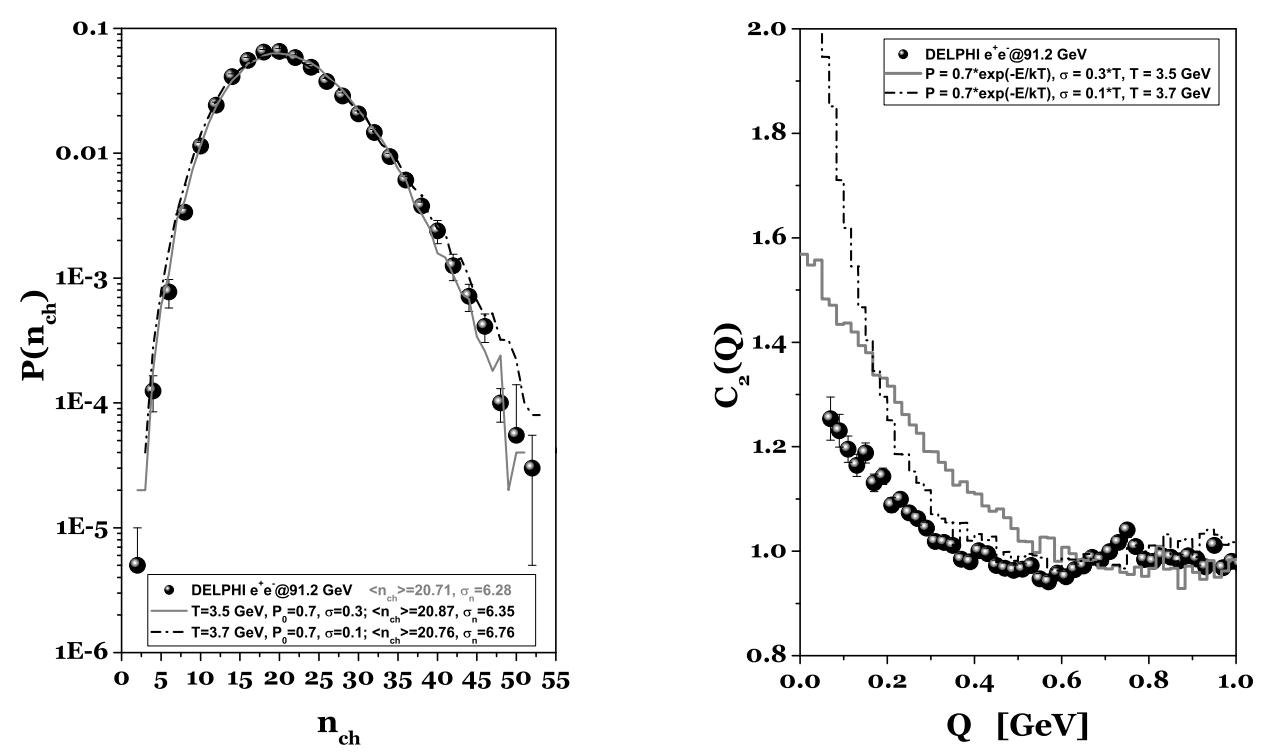

Fig. 2. Examples of confrontation with the experimental data [17]. Left panel: fit to charge multiplicity distribution. Right panel: results for $C_{2}\left(Q=\left|p_{1}-p_{2}\right|\right.$ ) correlation function (one dimensional phase space was used here only). Two different sets of parameters have been used. Notice that whereas they lead to essentially similar $P(n)$ the resulting $C_{2}(Q)$ are drastically different.

cles in each EEC can have (slightly) different energies from energy of the particle being its seed. To do it one must allow that each additional particle selected in point 2) above have energy $E_{i}^{(1)}$ selected from some distribution function peaked at $E_{1}^{(1)}, G\left(E_{1}^{(1)}-E_{i}^{(1)}\right)$, where the width of this distribution, $\sigma$, is another free parameter. $^{b}$

4) Repeat points 1) - 3) as long as there is enough energy left. Correct in every event for every energy-momentum nonconservation caused by the selection procedure adopted and assure that $N^{(+)}=N^{(-)}$.

As a result, in each event we get a number of EEC with particles of the same charge and (almost) the same energy, i.e. picture closely resembling classical clans of [10] (with no effects of statistics imposed, see Fig. 1). Our clans (containing now identical bosonic particles subjected to quantum statistics and therefore named quantum clans) are distributed in the same way as the particles being the seeds for EEC, i.e. according to Poisson distribution. With particles in each clan distributed according to geometrical distribution they lead therefore to the overall distribution in our Quantum Clan Model case being of the so called Pòlya-Aeppli type [16]. This distribution strongly resembles the Negative Binomial distributions obtained in the classical clan model [10] where particles in each clans were assumed to follow logarithmic distribution instead (with differences occurring for small multiplicities [ 16]). The first preliminary results presented in Fig. 2] are quite encouraging 
(especially when one remembers that so far effects of resonances and all kind of final state interactions to which $C_{2}$ is sensitive were neglected here). It remains now to be checked what two-body BEC functions for other components of the momentum differences and how they depend on the EEC parameters: $T, P_{0}$ and $\sigma$. So far the main outcome is suggestion that EEC's are among the possible explanations of the BEC effect, in which case BEC would provide us mainly with their characteristics. This should clear at least some of many apparently "strange" results obtained from BEC recently [18].

\section{Acknowledgment(s)}

Two of us (OU and GW) are grateful for support from the Hungarian Academy of Science and for the warm hospitality extended to them by organizers of the $4^{\text {th }}$ Budapest Winter School on Heavy Ion Physics, Dec. 1-3, 2004, Budapest, Hungary. Partial support of the Polish State Committee for Scientific Research (KBN) (grant 621/E-78/SPUB/CERN/P-03/DZ4/99 (GW)) is acknowledged.

\section{Notes}

a. It means then that from $C_{2}$ one gets not the size of the hadronizing source but only the size of the emitting cell, in [2] $R \sim 1 / \delta y$, cf. [13]. In the quantum field theoretical formulation of BEC this directly corresponds to the necessity of replacing delta functions in commutator relations by well-defined peaked functions introducing in this way the same dimensional scale to be obtained from the fits to data [14]. This fact was known even before but without any phenomenological consequences [15].

b. It reflects situation encountered in [14] where, as we have already mentioned before, the observed shape of $C_{2}(Q)$ was coming from the assumed shape of function replacing Dirac delta function in energy, i.e. introducing a smearing in energy.

\section{References}

1. Cf., for example, T. Csörgö, in Particle Production Spanning MeV and TeV Energies, eds. W.Kittel et al., NATO Science Series C, Vol. 554, Kluwer Acad. Pub. (2000), p. 203 hep-ph/0001233; W. Kittel, Acta Phys. Polon. B32 (2001) 3927; G. Alexander, Rep. Prog. Phys. 66 (2003) 481, and references therein.

2. T. Osada, M. Maruyama and F. Takagi, Phys. Rev. D59 (1999) 014024.

3. W. Zajc, Phys. Rev. D53 (1987) 3396; R.L. Ray, Phys. Rev. C57 (1998) 2352; J.G.Cramer, Event Simulation of High-Order Bose-Einstein and Coulomb Correlations, Univ. of Washington preprint, 1996, unpublished. 
4. L. Lönnblad and T. Sjöstrand, Eur. Phys. J. C2 (1998) 165; K. Fiałkowski, R. Wit and J. Wosiek, Phys. Rev. D58(1998) 094013; T.Wibig, Phys. Rev. D53 (1996) 3586. B. Andersson, Acta Phys. Polon. B29 (1998) 1885;

K. Geiger, J. Ellis, U. Heinz and U.A. Wiedemann, Phys. Rev. D61(2000) 054002.

5. K.J. Eskola, Nucl. Phys. $\mathbf{A 6 9 8}$ (2002) 78; S.A.Bass et al. Prog. Part. Nucl. 41 (1998) 225.

6. O.V. Utyuzh, G. Wilk and Z. Włodarczyk, Phys. Lett. B522 (2001) 273 and Acta Phys. Polon. B33(2002) 2681.

7. A. Białas and A. Krzywicki, Phys. Lett. B354, (1995) 134; H. Merlitz and D. Pelte, Z. Phys. A351(1995) 187 and A357 (1997) 175; U.A. Wiedemann et al., Phys. Rev. C56 (1997) R614; T. Csörgö and J. Zimányi, Phys. Rev. Lett. 80 (1998) 916 and Heavy Ion Phys. 9 (1999) 241.

8. W.J. Knox, Phys. Rev. D10 (1974) 65; E.H. De Groot and H. Satz, Nucl. Phys. B130 (1977) 257; J. Kripfganz, Acta Phys. Polon. B8(1977) 945; A.M. Cooper, O. Miyamura, A. Suzuki and K. Takahashi, Phys. Lett. B87 (1979) 393; F. Takagi, Prog. Theor. Phys. Suppl. 120 (1995) 201.

9. E.E. Purcell, Nature 178 (1956) 1447; A. Giovannini and H.B. Nielsen, Stimulated emission model for multiplicity fluctuations, The Niels Bohr Institute preprint, NBI-HE-73-17 (unpublished); Stimulated emission effect on multiplicity distribution in: Proc. of the IV Int. Symp. on Multip.

Hadrodynamics, Pavia 1973, Eds. F. Duimio, A. Giovannini and S. Ratii, p. 538.

10. A. Giovannini and L. Van Hove, Z. Phys. C30 (1986) 381; see also: P. Carruthers and C.S. Shih, Int. J. Mod. Phys. A2 (1986) 1447.

11. M. Biyajima, N. Suzuki, G. Wilk and Z. Włodarczyk, Phys. Lett. B386 (1996) 297.

12. F.S. Navarra, O.V. Utyuzh, G. Wilk and Z. Włodarczyk, Phys. Rev. D67 (2003) 114002.

13. W.A. Zajc, A pedestrian's guide to interferometry, in Particle Production in Highly Excited Matter, eds. H.H.Gutbrod and J.Rafelski, Plenum Press, New York 1993, p. 435.

14. G.A. Kozlov, O.V. Utyuzh and G. Wilk, Phys. Rev. C68 (2003) 024901; Ukr. J. Phys. 48 (2003) 1313.

15. K. Zalewski, Lecture Notes in Physics 539(2000) 291.

16. J. Finkelstein, Phys. Rev. D37 (1988) 2446; Ding-wei Huang, Phys. Rev. D58 (1998) 017501.

17. P.Abreu et al. (DELPHI Collab.), Phys. Lett. B286 (1992) 201; Phys. Lett. B247 (1990) 137.

18. J.T. Mitchell, J. Phys. G30 (2004) S819; H. Appelhäuser, ibid., S935. 